\section{Tratamiento de mixoma odontogénico en maxilar superior. Reporte de un caso}

\section{Treatment of odontogenic myxoma in upper maxillary. A case report}

\section{Resumen}

El mixoma odontogénico es una neoplasia benigna, localmente agresiva, con una baja prevalencia (frecuencia del $2 \%$ al $5 \%$ de los tumores odontogénicos) en los maxilares. Este tumor a menudo se presenta como una lesión expansiva asintomática sin cambios nerviosos sensoriales. El objetivo del presente trabajo es detallar el caso de un paciente masculino de 42 años quien acude al servicio de Cirugía Bucal y Maxilofacial del Hospital Nacional Hipólito Unanue por presentar una tumoración de crecimiento lento en maxilar superior, que fue aumentando de tamaño con el tiempo y desplazando órganos dentarios, y que se extiende al paladar duro y parte de paladar blando, el diagnóstico anatomopatológico fue de mixoma odontogénico y recibió un tratamiento radical (hemimaxilectomia) por la naturaleza agresiva del tumor. En este trabajo discutimos la presentación del mixoma odontogénico en el maxilar superior, los enfoques de tratamiento; detallando la importancia de plantear alternativas de tratamiento que permitan la reconstrucción inmediata generando la menor morbilidad posible.

Palabras clave: Mixoma; Colgajos quirúrgicos; Procedimientos quirúrgicos reconstructivos (fuente: DeCS BIREME).

\begin{abstract}
Odontogenic myxoma is a locally aggressive benign neoplasm with a low prevalence (frequency $2 \%$ to $5 \%$ of odontogenic tumors) in the jaws. This tumor often presents as an asymptomatic expansive lesion without sensory nerve changes. The aim of this study is to detail the case of a 42-year-old male patient who attends the Oral and Maxillofacial Surgery service of the Hipólito Unanue National Hospital due to a slow-growing tumor in the upper jaw, which increased in size over time and displacing dental organs, and extending to the hard palate and part of the soft palate, the pathological diagnosis was odontogenic myxoma and received radical treatment (hemimaxilectomy) due to the aggressive nature of the tumor. In this work we discuss the presentation of odontogenic myxoma in the maxilla, treatment approaches; detailing the importance of proposing treatment alternatives that allow immediate reconstruction generating the least possible morbidity.
\end{abstract}

Keywords: Myxoma; Surgical flap; Reconstructive surgical procedures (source: $\mathrm{MeSH}$ NLM).

\section{Caso Clínico}

David Erik Moreno Villalobos ${ }^{1,2, a, b}$, Arturo Rodríguez

Flores $^{1, a, b}$, Diana Carolina Vargas Rojas ${ }^{1,2, a}$

${ }^{1}$ Hospital Nacional Hipólito Unanue, Lima, Perú.

${ }^{2}$ Universidad Nacional Mayor de San Marcos, Facultad de Odontología, Lima, Perú.

a Cirujano Dentista.

${ }^{\text {b }}$ Especialidad en Cirugía Bucal y Maxilofacial.

\section{Correspondencia:}

Diana Carolina Vargas Rojas: dcvr1991@gmail.com

Jr. Jorge Chávez 269 - Breña

ORCID: 0000-0002-4619-3317

\section{Coautores:}

David Erik Moreno Villalobos: david.moreno.v@hotmail.com ORCID: 0000-0003-4348-7042

Arturo Rodríguez Flores: aarturor@hotmail.com

ORCID: 0000-0003-0528-7314

\section{Editora:}

Dahiana Alfaro Carballido

Universidad de San Martín de Porres, Perú.

Conflicto de intereses: los autores declaran que no existe conflicto de interés.

Financiamiento: autofinanciado.

Recibido: 25/08/20

Aceptado: $21 / 11 / 20$

Publicado: 01/04/21 


\section{Introducción}

El mixoma odontogénico (MO), neoplasia odontogénica benigna de origen mesenquimal, es el tercer tumor odontogénico más común (2-5\%), dos tercios se localizan en la mandíbula y un tercio al maxilar superior. Hasta la actualidad existe controversia sobre la patogénesis de estas lesiones ${ }^{~}$. Anteriormente se clasificó en dos variantes: centrales y periféricos. Las lesiones periféricas son menos agresivas y encapsuladas; por el contrario, los centrales son generalmente tumores no encapsulados con capacidad infiltrativa en el hueso medular adyacente ${ }^{2,3}$. Histológicamente, los mixomas muestran un delicado estroma mucoide fibroso, esto se debe a la presencia de células mesenquimales indiferenciadas que muestran diferenciación fibroblástica ${ }^{4,5}$. Cuando el estroma de colágeno denso es evidente, el término fibromixoma / mixofibroma puede ser usado. Debido a este patrón dual, se cree que el mixoma odontogénico es precedido de fibroma odontogénico ${ }^{6}$.

El MO maxilar es menos frecuente y se comporta de manera más agresiva que en la mandíbula, porque se propaga a través del seno maxilar, causando aflojamiento y desplazamiento del diente $y$, con menos frecuencia, reabsorción radicular; exoftalmos y obstrucción nasal en casos severos ${ }^{7}$.

El presente informe destaca una entidad rara de tumor odontogénico, con un diagnóstico diferencial de mixoma odontogénico central en maxilar superior en un paciente adulto. Debido al patrón agresivo de la lesión se optó por realizar un tratamiento radical (hemimaxilectomia); y a la vez realizar una reconstrucción inmediata.

El objetivo del presente trabajo es proporcionar información que puede mejorar la precisión del diagnóstico, permitiendo a los cirujanos dentistas y maxilofaciales guiar a proponer alternativas de tratamiento para optimizar los resultados clínicos.

\section{Reporte del caso}

Diagnóstico y etiología. Paciente varón de 42 años de edad, de ocupación obrero, sin antecedentes médicos de importancia. Acude a la consulta por presentar un tumor en su boca que va creciendo desde hace 5 años, posterior a una exodoncia, A la evaluación clínica especializada extraoral; presenta aumento de volumen en región geniana de lado derecho, borramiento del surco nasogeniano, piel que lo cubre de características normales y sensibilidad conservada. Al examen intraoral; se evidencia una lesión de tipo tumor redondeado (de 3 $\mathrm{cm} \times 2 \mathrm{~cm} \times 1 \mathrm{~cm}$ ), base amplia, de color similar al de la mucosa adyacente, superficie lisa con indentaciones, no móvil, de bordes definidos, que se localiza en reborde alveolar superior con extensión a fondo de surco y paladar, cruzando la línea media, indurada, no dolorosa a la palpación, desplaza órganos dentarios $(13,14,17)$, con movilidad de $2^{\circ}$ grado (Figura 1). Se solicita radiografía panorámica y tomografía cone beam (Figuras 2 y 3 ). El

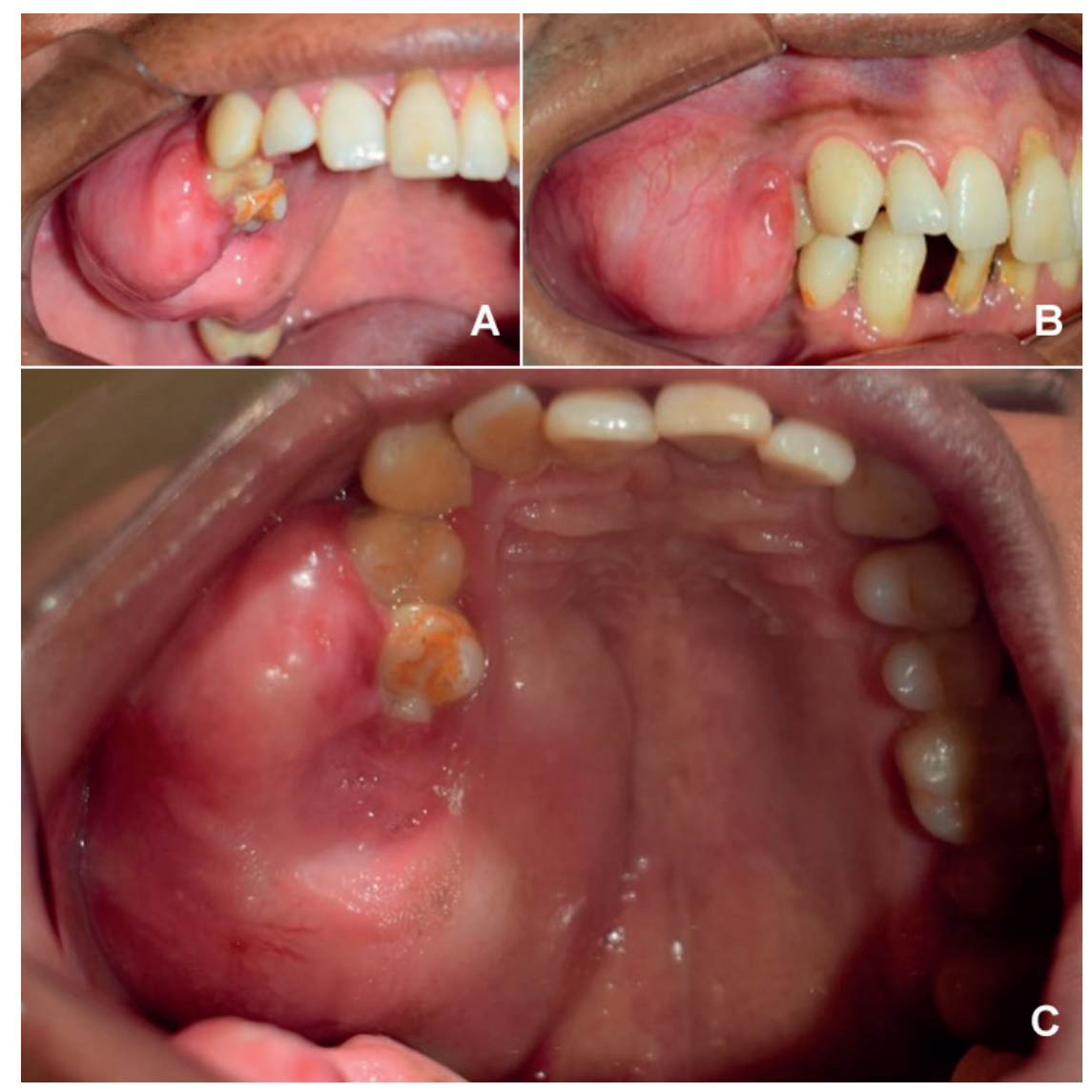

Figura 1. Fotografías clínicas preoperatorias: intraorales (A, B, C) 


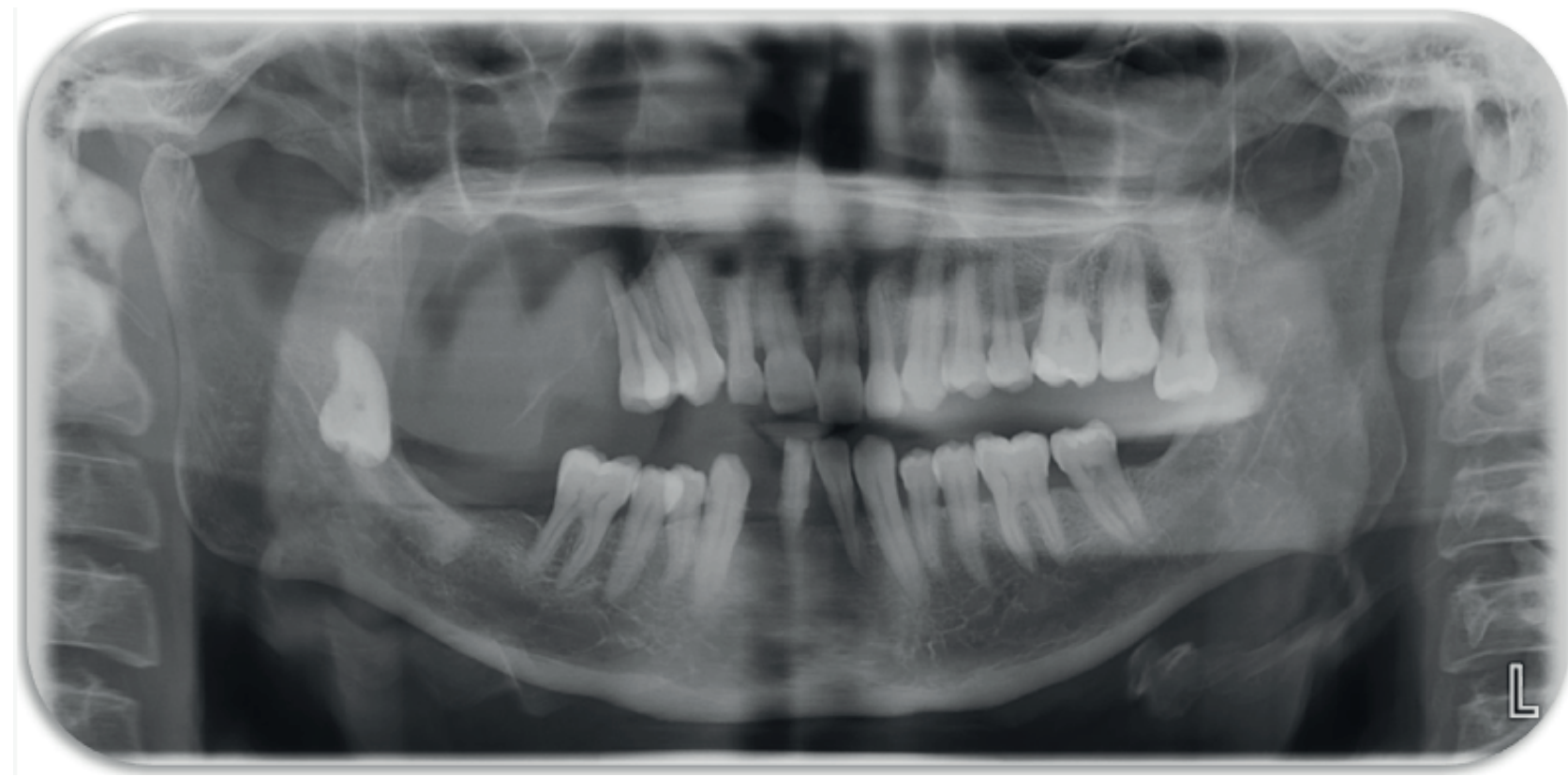

Figura 2. Radiografía panorámica: muestra imagen radiolúcida con bordes poco definidos, no causa reabsorción radicular y desplaza piezas dentarias. Localizado en maxilar superior ocupando el seno maxilar derecho
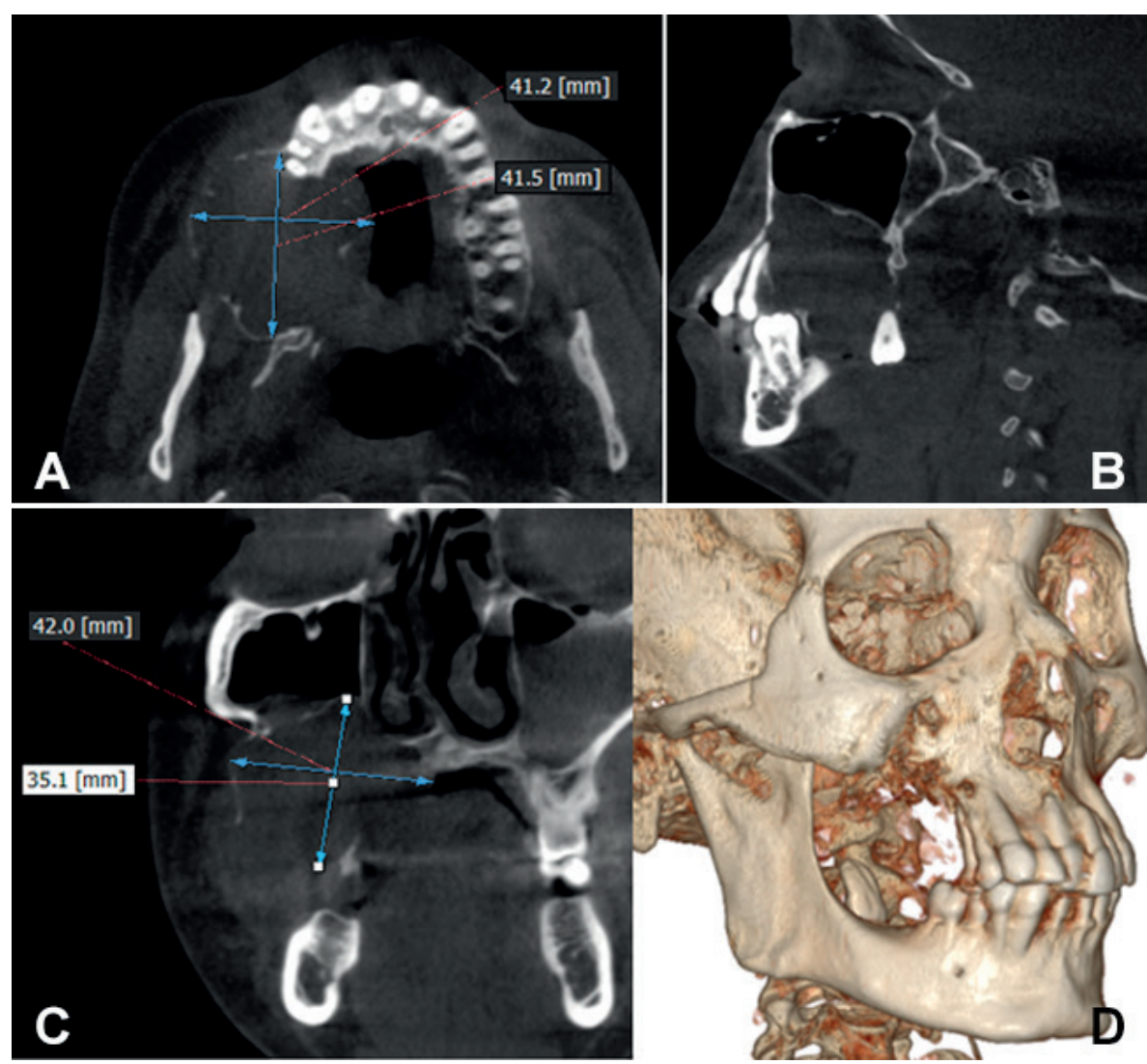

Figura 3. Tomografía cone beam: muestra imagen isodensa que compromete maxilar superior derecho, invade seno maxilar, mide $4 \times 4 \times 3 \mathrm{~cm}$ y perfora cortical vestibular y palatina (A, B, C). En la imagen de reconstrucción 3D se evidencia la destrucción ósea (D)

resultado anatomopatológico informó mixoma odontogénico en maxilar superior derecho (Figura 4).

Selección del tratamiento quirúrgico. Confirmado el diagnóstico se solicita el consentimiento informado al paciente; y dado el comportamiento agresivo de la lesión, se programa la intervención quirúrgica que consistió en: hemimaxilectomia con márgenes de seguridad mediante un abordaje de weber-ferguson y reconstrucción inmediata del defecto con rotación de colgajo temporal e injerto de fascia de temporal (Figuras 5 y 6 ); para lo que 


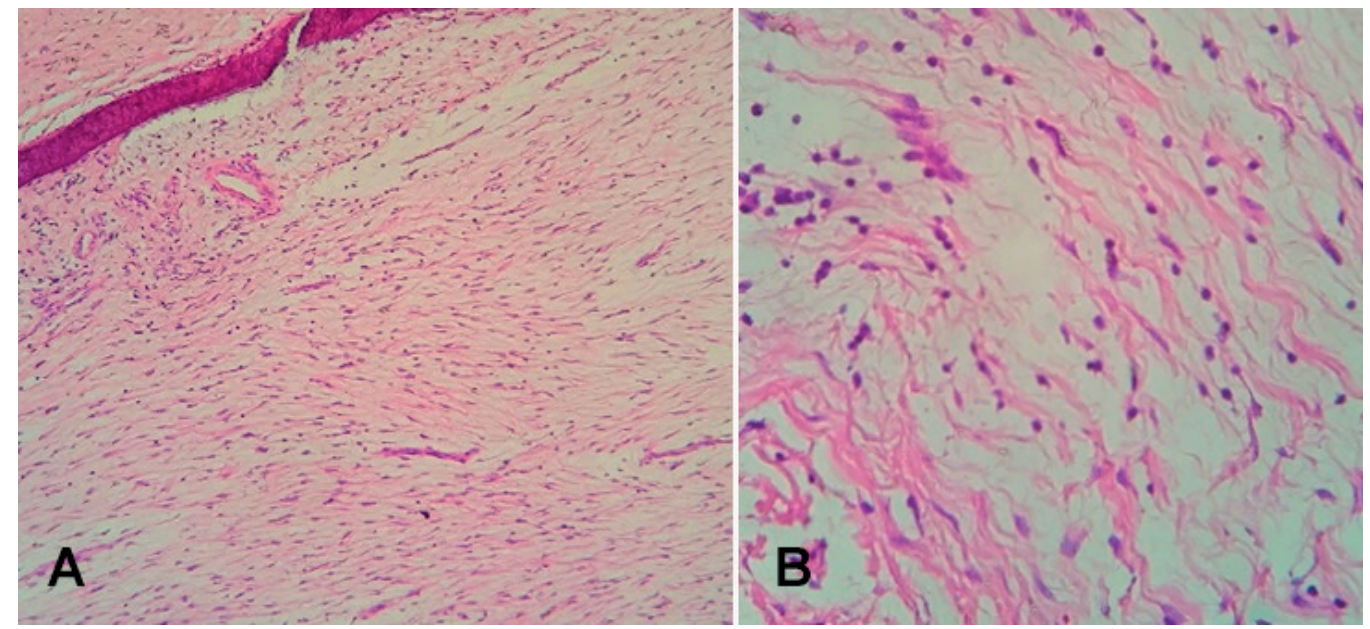

Figura 4. Muestra histológica. A. (100x), B. (400x) (Tinción HE) Se logra evidenciar células redondeadas, fusiformes, orientadas al azar con procesos citoplasmáticos largos ligeramente eosinófilos; dispersados uniformemente en un estroma de sustancia fundamental

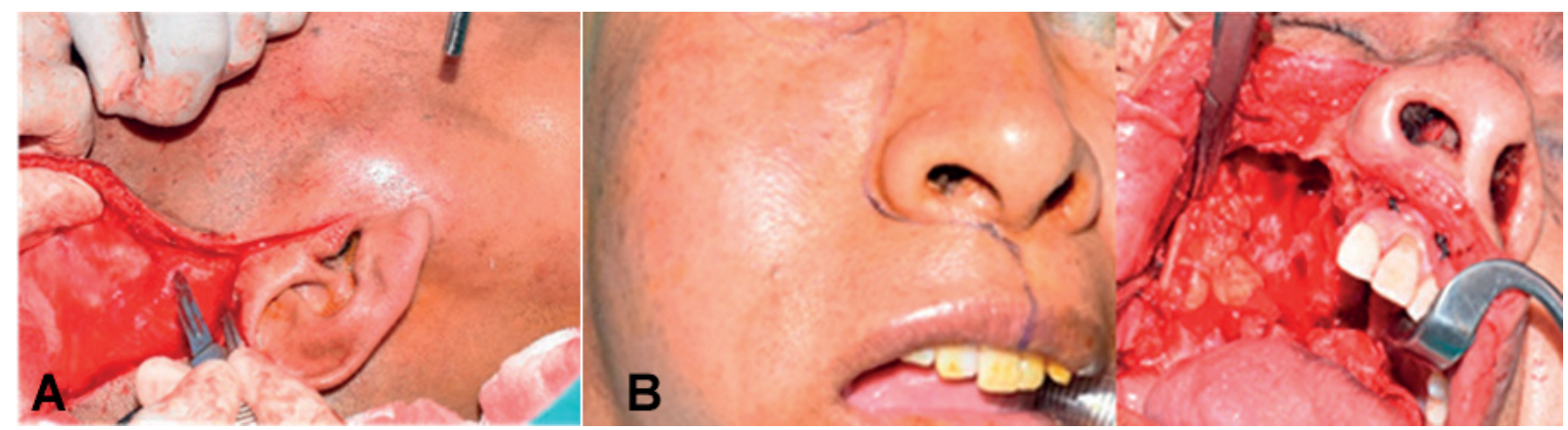

Figura 5. Abordajes operatorios: coronal (A) y Weber-Ferguson (B)
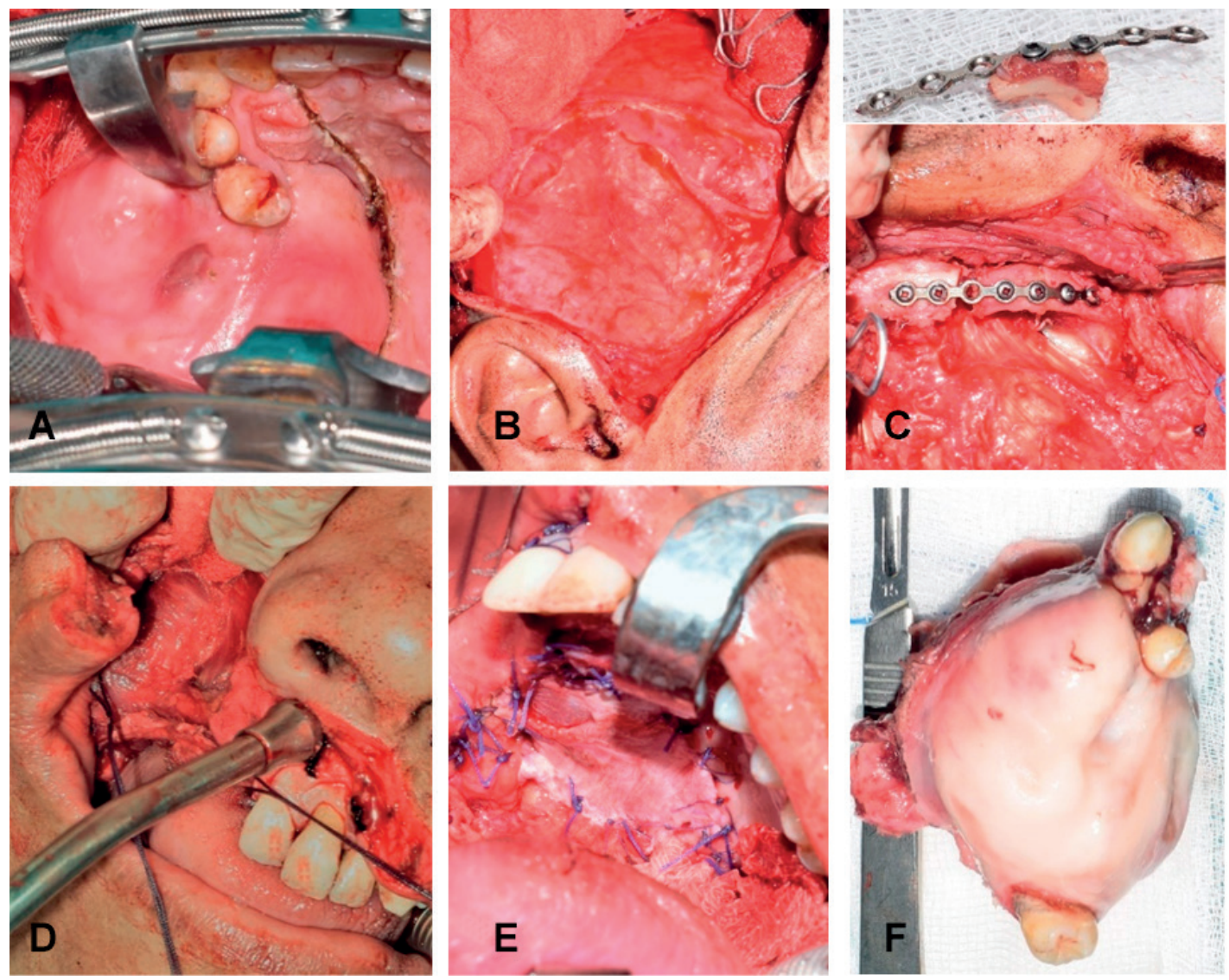

Figura 6. Procedimiento quirúrgico: resección de la lesión (A), cosecha de injerto de fascia temporal (B). Osteotomía de arco cigomático (C). Rotación de musculo temporal al defecto (D). Sutura y cierre del defecto (E). Pieza operatoria (F) 
se realiza abordaje coronal de lado derecho con extensión preauricular, se toma el injerto de fascia de temporal, se realiza disección de fascículo anterior del musculo temporal, osteotomía a nivel de arco cigomático derecho para facilitar el paso del musculo temporal, se fija el musculo con puntos de sutura al defecto, se reposiciona el arco cigomático con material de osteosíntesis, se rota la almohadilla de grasa bucal y se fija con el injerto de fascia del temporal para cerrar todo el defecto. Al paciente se le indica la colocación de sonda nasogástrica para posterior alimentación y asegurar adecuada cicatrización de la herida operatoria (Figuras 5 y 6). El tratamiento farmacológico se complementa con antibioticoterapia endovenosa por 7 días, suplementos vitamínicos y terapia del dolor.
Resultados del tratamiento. Durante los controles post operatorios inmediatos no se evidencio comunicación oro nasal. Por lo que se progresa a una dieta blanda atraumatica. A nivel de la región temporal se pudo notar una asimetría, que se vuelve poco notoria con el crecimiento del cabello. A los 20 días posteriores a la cirugía, ya se pudo evidenciar la mucosa reparada en toda la extensión del defecto (Figura 7).

Los controles a largo plazo, transcurrieron sin mayores problemas, actualmente la evolución es favorable (Figura 8), el paciente acude a controles periódicos dado la naturaleza de la lesión y el grado de recurrencia que pueda presentar.
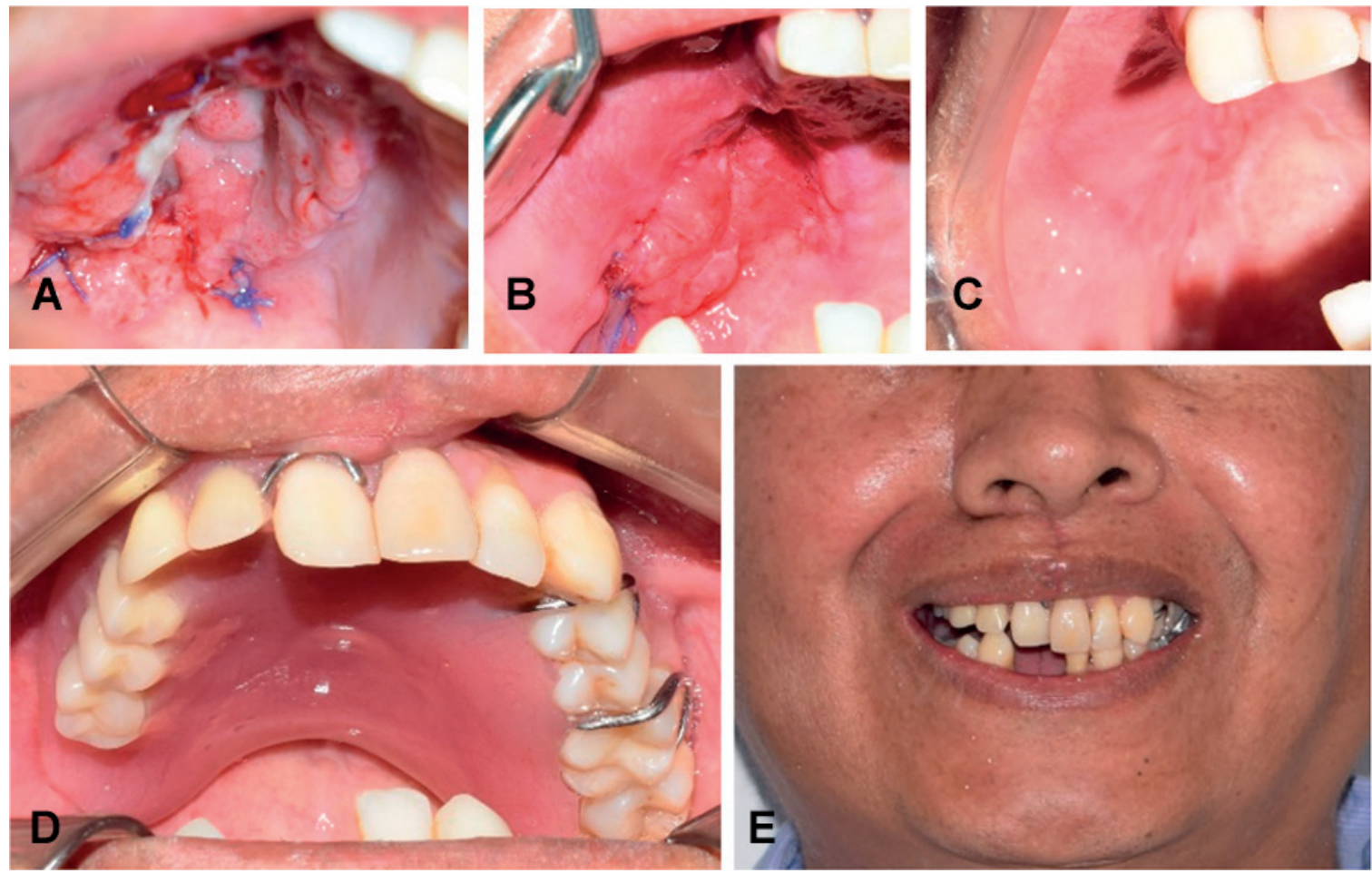

Figura 7. Controles postoperatorios: a los 5 días (A), 15 días (B), 30 días (C). Colocación de prótesis convencional a los 2 meses post operatorio ( $D$ y $E$ ).
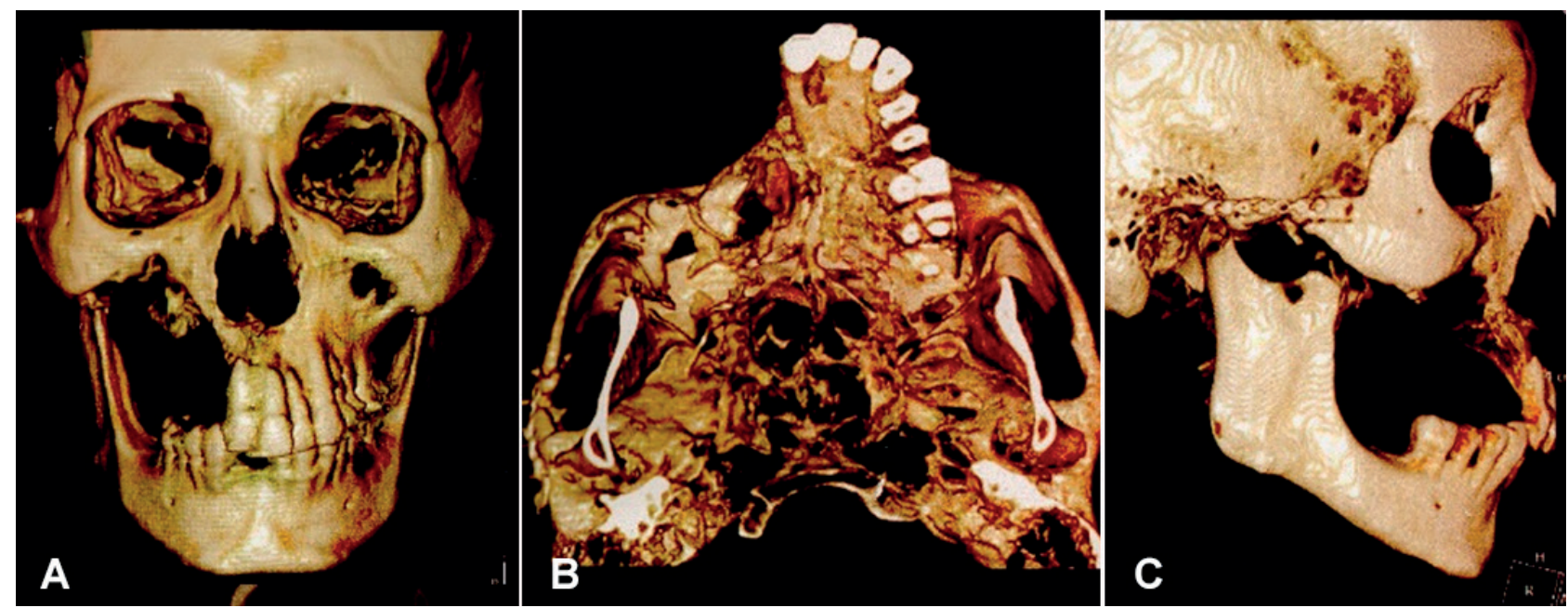

Figura 8. Tomografía cone-beam post operatorio 6 meses. A. Reconstrucción 3D frontal. B. Reconstrucción 3D axial. C. Reconstrucción 3D lateral derecha 


\section{Discusión}

El MO a menudo se comporta de manera localmente agresiva e infiltrante. Ha sido catalogado como el tercer tumor odontogénico más frecuente después del odontoma y el ameloblastoma ${ }^{8}$.

Chrcanovic y Gomez ${ }^{8}$ informaron que las lesiones fueron más prevalentes en la mandíbula en comparación del maxilar superior y en la región posterior en comparación con la región anterior. Reportaron que 86 de 1261 lesiones $(6,8 \%)$ cruzaron la línea media de los maxilares, y el seno maxilar se vio significativamente afectado en 163 de 344 lesiones $(47,4 \%)$ ubicadas en el maxilar y 18 lesiones alcanzaron la apófisis coronoides. Mientras que Tavakoli y Williamson ${ }^{9}$ informaron que las lesiones maxilares ocurren por igual en todas las áreas y con frecuencia erosionan el seno, a menudo cruzando la línea media y hacia la cavidad sinusal opuesta.

La presentación radiográfica del mixoma es variable. El tumor puede presentarse como una lesión radiotransparente unilocular o multilocular, la presencia de tabiques angulares da una apariencia de "panal de abeja" o "burbuja de jabón". Los bordes pueden estar bien definidos o mal definidos ${ }^{10,11}$. El diagnóstico diferencial incluye ameloblastoma, fibroma odontogénico, quiste dentígero, queratoquiste odontogénico y tumores no odontogénicos como granuloma central de células gigantes, fibroma osificante, hemangioma y osteosarcoma ${ }^{12}$. Histológicamente el $\mathrm{MO}$ es idéntico a la papila dental en desarrollo, folículo dental normal o hiperplásico, por esta razón es importante la correlación clínica e imagenológica ${ }^{1}$.

Existe una controversia en la literatura sobre la modalidad de tratamiento más apropiada para el MO. Pahl et al. ${ }^{13}$ en su estudio ya informaban que las tasas de recurrencia relativamente altas con el legrado y la enucleación pueden atribuirse a la falta de encapsulación y a la sutil invasión local de neoplasias entre el hueso esponjoso más allá de los márgenes radiográficamente visibles.

Chrcanovic y Gomez ${ }^{8}$ también reportaron que el legrado fue el tratamiento con la tasa más alta de recurrencia, seguido de enucleación. El legrado adicional después de la enucleación tuvo una tasa de recurrencia similar en comparación con la enucleación sola, pero el rendimiento de la osteotomía periférica disminuyó la tasa de recurrencia. El tratamiento por resección, independientemente de si era marginal o segmentario, tuvo la tasa de recurrencia más baja; concluyen que a pesar de que la resección mostró las tasas de recurrencia más bajas, los cirujanos deben tener en cuenta que la enucleación tumoral seguida de ostectomía periférica podría considerarse como opción de tratamiento debido a la menor morbilidad asociada. Así mismo sugieren en su estudio que los casos que comprometen segmentos de la mandíbula o maxilar en toda su extensión caudocefálica con adelgazamiento cortical extenso y grandes áreas de perforación cortical deben tratarse con resección segmentaria ${ }^{8}$.
Boffano et al. ${ }^{14}$ en su estudio también nos detalla que en el caso de elegir o realizar un tratamiento radical; recomiendan la reconstrucción inmediata después de la resección, disminuyendo así la morbilidad originada por la naturaleza del tratamiento ${ }^{14}$.

Jategoankar et al. ${ }^{15}$ realizaron un estudio sobre defectos palatomaxilares, en el que describen que el complejo palatomaxilar representa una unidad estructural y funcional del rostro, la cirugía que compromete este complejo puede resultar en una morbilidad funcional. Así que la reconstrucción de estas estructuras de la cara media es primordial y representan un desafío para el cirujano reconstructivo. Los autores describen diferentes alternativas de reconstrucción los que incluyen aparatos protésicos, colgajos locales y/o regionales y transferencia de colgajo microvascular.

Brown et al. ${ }^{16}$ presento su clasificación basada en los componentes vertical y horizontal del defecto; el defecto de nuestro paciente encaja en el grupo de defectos de tipo IIB. Jategoankar en su estudio recomiendan la reconstrucción de estos defectos por medio de colgajo microvascular que incluyan tejido óseo y músculo ${ }^{15}$.

Cordeiro et al. ${ }^{17}$ plantean que para los defectos que no requieren reconstrucción con colgajos microvasculares se puede utilizar injertos óseos libres y/o musculo temporal transpuestos anteriormente, para lo que se debe osteotomizar el arco cigomático para permitir el paso del músculo. El tratamiento de reconstrucción de nuestro paciente se basó en esta propuesta, teniendo en cuenta que en el presente caso solo se usó el colgajo temporal transpuesto en el defecto.

Se concluye que el tratamiento con resección amplia para el MO extenso es recomendable debido al alto riesgo de recurrencia local. Realizamos una reconstrucción inmediata que implica el cierre primario del defecto con rotación de colgajo temporal, rotación de almohadilla de grasa bucal y el uso de injerto de fascia temporal autógeno para reconstruir estructuras vitales y evitar la comunicación oro nasal; así garantizamos al paciente menor morbilidad y posteriormente poder llegar a confeccionar una prótesis convencional para ayudar en la masticación, el habla y para proporcionar apoyo facial.

Si bien es sabido que el tratamiento para estas lesiones se encuentra en un espectro desde la enucleación hasta la resección radical; Los procedimientos quirúrgicos más conservadores se asocian con una mayor probabilidad de recurrencia de MO. Por tal motivo aconsejamos un tratamiento radical siempre en cuando podamos también asegurar la menor morbilidad generada al paciente con técnicas quirúrgicas de reconstrucción inmediata.

\section{Agradecimiento}

Se agradece al Servicio de cirugía bucal y maxilofacial y a todo el equipo quirúrgico participante en el desarrollo de este caso clínico. 


\section{Referencias bibliográficas}

1. El-Naggar A, Chan J, Grandis J, Takata T and Slootweg P. WHO Classification of Head and Neck Tumours. $4^{\text {th }}$ Edition. Lyon: International Agency for Research on Cancer; 2017.

2. Shah A, Lone P, Latoo S, Ahmed I, Malik A, Hassan S, et al. Odontogenic myxoma of the maxilla: A report of a rare case and review on histogenetic and diagnostic concepts. Natl J Maxillofac Surg [revista en Internet] 2011 Julio [acceso 18 de diciembre 2020]; 2(2):189-95. Disponible en: https://www.njms.in/text.asp?2011/2/2/189/94480.

3. Mehendiratta M, Rehani S, Solomon MC. The histological spectrum of myxoma, myxofibroma/fibromyxoma and odontogenic fibroma - "Chicken and Egg situation". IOSR Journal of Dental and Medical Sciences (JDMS) [revista en Internet] 2012 setiembre - octubre [acceso 25 de enero 2020]; 1(2):3-5. DOI: 10.9790/08530120305. Disponible en: https://www.researchgate.net/ publication/315297637_The_Histological_Spectrum_ of_Myxoma_Myxofibroma_Fibromyxoma_and_Odontogenic_Fibroma-_A_Chicken_And_Egg_Situation.

4. Kawase-Koga Y, Saijo H, Hoshi K, Takato T, Mori Y. Surgical management of odontogenic myxoma: a case report and review of the literature. BMC Res Notes [revista en Internet] 2014 Abril [acceso 16 de enero 2020]; 7(214). Disponible en: https://doi.org/10.1186/17560500-7-214.

5. Manne RK, Kumar VS, Sarath PV, Anumula L, Mundlapudi S, Tanikonda R. Odontogenic Myxoma of the Mandible. Case Rep Dent. [revista en Internet] 2012 Julio [acceso 02 de febrero 2020], 2012. Disponible en: https://doi.org/10.1155/2012/214704.

6. Godishala SR, Naag S, Bahl S, Priyadarshini E. Odontogenic myxoma: A causality dilemma - Report of a nonpareil case and review of literature. J Oral Maxillofac Pathol [revista en Internet] 2018 Enero [acceso 22 de enero 2020] ;22(4):2-6. Disponible en: http://www.jomfp.in/ article. asp? issn=0973-029X; year $=2018$; volume $=22$; issue $=4$; page $=2$; epage $=6$; aulast $=$ Godishala .

7. Vijayabanu B, Sreeja C, Bharath N, Aesha I, Kannan VS, Devi M. Odontogenic myxoma of maxilla: A rare presentation in an elderly female. J Pharm Bioallied Sci [revista en Internet] 2015 Agosto [acceso 10 de abril 2020]; 7(6):759-62. Disponible en: https://www.ncbi.nlm.nih. gov/pmc/articles/PMC4606704/.

8. Chrcanovic BR, Gomez RS. Odontogenic myxoma: An updated analysis of 1,692 cases reported in the literature. Oral Dis[revista en Internet] 2019 Abril [acceso 24 de enero 2020]; 25(3):676-683. Disponible en: https://doi. org/10.1111/odi.12875.
9. Tavakoli M, Williamson R. Odontogenic myxomas: what is the ideal treatment? BMJ Case Rep [revista en Internet]. 2019 abril [acceso 24 de enero del 2020]; 12(5). Disponible en: http://dx.doi.org/10.1136/bcr2018-228540.

10. Regezi JA, Sciubba JJ, Jordan RCK. Oral pathology: clinical pathological correlations. $4^{\text {th }}$ ed. Philadelphia: Saunders; 2003.

11. Noffke CE, Raubenheimer EJ, Chabikuli NJ, Bouckaert MM. Odontogenic myxoma: review of the literature and report of 30 cases from South Africa. Oral Surg Oral Med Oral Pathol Oral Radiol Endod [revista en Internet] 2007 Julio [acceso 25 de enero 2020]; 104(1):101-109. DOI: $10.1016 /$ j.tripleo.2007.01.026. Disponible en: https://pubmed.ncbi.nlm.nih.gov/17507265/.

12. Marx RE, Stern D. Oral and maxillofacial pathology: a rationale for diagnosis and treatment. $2^{\text {nd }} \mathrm{Ed}$. Hanover Park: Quintessence Publishing Co; 2012.

13. Pahl S, Henn W, Binger T, Stein U, Remberger K. Malignant odontogenic myxoma of the maxilla: case with cytogenetic confirmation. J Laryngol Otol [revista en Internet] 2000 Julio [acceso 22 de enero 2020]; 114(7):533535. DOI: 10.1258/0022215001906075. Disponible en: https://pubmed.ncbi.nlm.nih.gov/10992937/.

14. Boffano P, Gallesio C, Barreca A, Bianchi FA, Garzino-Demo P, Roccia F. Surgical treatment of odontogenic myxoma. J Craniofac Surg [revista en Internet] 2011 May [acceso 26 de enero 2020]; 22(3):982-987. DOI: 10.1097/SCS.0b013e3182101400. Disponible en: https://pubmed.ncbi.nlm.nih.gov/21558907/.

15. Jategaonkar A, Kaul V, Lee E and Genden E. Surgery of the Palatomaxillary Structure. Semin Plast Surg [revista en internet] 2020 May [acceso 24 de Julio 2020]; 34(02): 071-076. Disponible en: https://doi.org/10.1055/s-0040-1709430.

16. Brown JS, Rogers SN, McNally DN, Boyle M. A modified classification for the maxillectomy defect. Head Neck [revista en internet] 2000 Jan [acceso 22 de Julio 2020]; 22(1):17-26. Disponible en: https://doi.org/10.1002/ (sici) 1097-0347(200001)22:1<17::aid-hed4>3.0.co;2-2.

17. Cordeiro P, Santamaria E. A classification system and algorithm for reconstruction of maxillectomy and midfacial defects. Plast Reconstruct Surg. [revista en internet] 2000 Jun [acceso 24 de Julio 2020]; 105(7), 2331-2348. Disponible en https://doi.org/10.1097/00006534200006000-00004. 